\title{
Colecistitis y Colelitiasis en los niños
}

La colecistitis y colelitiasis en los niños. se describen con relativa poca frecuencia en la literatura mundial y casi siempre asociadas a otra patología, como avemia hemolítica. Desde la descripción de Gibson, en 1734, hasta hoy día, así parecen demostrarlo. En Chile, las colecistopatías representan en los servicios quirúrgicos de adultos una incidencia importante, que se extiende también a los niños.

\section{MATERIAL Y METODO}

Se revisan las historias clínicas de 251 enfermos ingresados con los diagnósticos de $\mathrm{Co}$ lecistitis $y / o$ Colelitiasis al Servicio de Cirugía Infantil del Hospital Roberto del Río, entre los años 1962 y 1975.

231 enfermos fueron tratados quirúrgicamente y 20 enfermos no fueron intervenidos por tratarse en $\mathbf{9}$ de ellos de cólicos biliares, que no volvieron a consultar, y 11 eran cuadros médico-quirúrgicos, en que durante los exámenes se descartó el diagnóstíco de Colecistopatías: 2 hepatitis, 2 pancreatitis, 2 apendicitis, 1 de torsión de epiplon y 1 tabique vesicular, 3 niños con colecistografias nega. tivas.

\footnotetext{
* Servicio de Cirugia Infantil, Hospital Roberto del Río.

* Servicio de Anatomía Patologica. Hospital Roberto del $R i$

*" Servicio de Radiología. Hospital Roberto del Rio,
}

Rev. Chilena de Pediatría, Vol. 47, No 2, 1976

Dres. Iaime Cerda Sepúlveda * Fernanc'o Torres Kay, Julio Aguirre Fuentes,* Gustavo Aldunate Noel,* L. David Mirkin W** y Carlos Vildósola San Martín.***

\section{SEXO Y EDAD}

En nuestra serie hay un claro predominio del sexo femenino $(70,9 \%)$ y sólo en el grupo de menores de 6 años predominan los varones $(83,0 \%$ ) (Figura No 1).

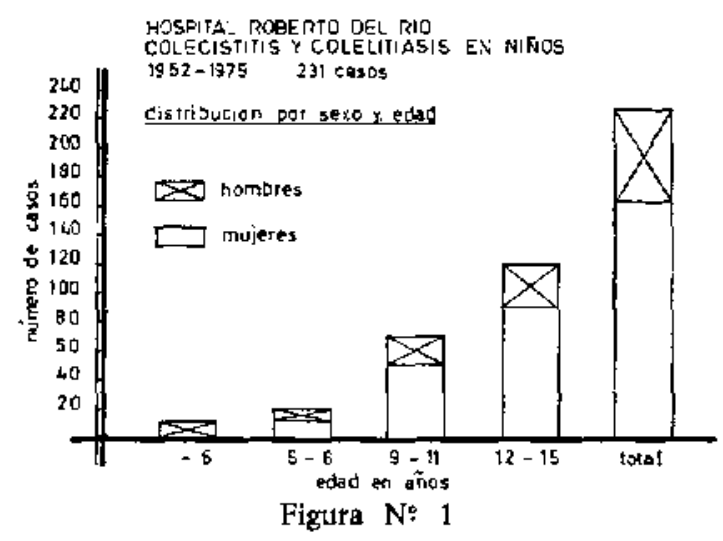

El grupo etario más frecuente es el de 12 a 15 años con 123 enfermos (76,0\%), cifra que nos parece disminuida, ya que algunos enfermos de este grupo consultan y se operan en servicios de adultos. Un $79,5 \%$ de las mujeres son impúberes.

\section{SINTOMATOLOGIA}

Hemos dividido las colecistitis agudas de las crónicas por considerar que hay diferencias claras en la sintomatología (Tabla No 1).

Colecistitis aguda. La encontramos en 126 casos y se caracteriza en los niños, por ser 
un cuadro violento, de dolor abdominal intenso de tipo cólico localizado en el hipocondrio derecho, sin irradiacion especial y que persiste en el mismo lugar. Se acompaña frecuentemente de vómitos $(84,6 \%)$ y fiebre $(58,4 \%)$. Sólo en un tercio de los casos $(35,3 \%)$ hay antecedentes de intolerancia selectiva por alimentos grasos y en la mitad de ellos crisis dolorosas anteriores $(50,7 \%)$.

La ictericia clínica es rara $(15,3 \%)$ y generalmente no hay gran compromiso del estado general $(10,7 \%)$.

El examen abdominal destaca la comprobación del dolor localizado en el hipocondrio derecho, y una vesícula palpable en la mitad de los casos $(49,2 \%)$ y la presencia de un plastrón vesicular en un $16,9 \%$. Signos francos de irritación peritoneal sólo en un 10,7\%.

El diagnóstico clínico es relativamente característico en las primeras horas del ingreso, lo que hace que estos enfermos se intervengan quirúrgicamente en forma de urgencia relativa (menos de 72 horas).

Colecistitis crónica. Se encuentra en $105 \mathrm{ni}$ ños y se caracteriza por tener frecuentemente antecedentes de crisis dolorosas de tipo cólico en el hipocondrio derecho $(74,6 \%)$; de dispepsia selectiva por las grasas en un tercio de los casos $(31,6 \%)$ y generalmente de curso insidioso, sin compromiso del estado general, ni fiebre.

El dolor en el hipocondrio derecho, siempre estuvo presente en el momento del ingreso $(98,7 \%)$ y en la mitad de los casos tienen vómitos $(46,5 \%)$. Vesícula palpable sólo en la cuarta parte de los niños $(25,3 \%)$.

La falta de compromiso del estado general o signos peritoneales, permiten realizar algunos exámenes importantes, especialmente la colecistografía.

En los niños menores de 6 años no hay antecedentes previos de dispepsia biliar o $c 6$. licos biliares, siendo el primer episodio de dolor intenso localizado en el hipocondrio derecho el motivo de consulta, acompañándose de fiebre $(71,4 \%)$, vómitos $(66,6 \%)$, ictericia, mal estado general, signos peritoneales y diarrea con mayor frecuencia. En general, el cuadro clínico indica mayor compromiso y gravedad, predominando las colecistitis tíficas y alitiásicas (Tabla № 2).

Tabla N: 1

\section{COLECISTITIS Y COLELITIASIS EN NINOS}

1962 - 1975 - 231 casos. Hospital Roberto del Río Sintomatologia

\begin{tabular}{lrr}
\hline & Agudas & Crónicas \\
\hline & & \\
Dolor abdominal & $100,0 \%$ & $98,7 \%$ \\
Vómitos & $84,6 \%$ & $46,8 \%$ \\
Crisis anteriores & $50,7 \%$ & $74,6 \%$ \\
Fiebre & $\mathbf{5 8 , 4} \%$ & $7,5 \%$ \\
Dispepsia & $35,3 \%$ & $31,6 \%$ \\
Ictericia & $\mathbf{1 5 , 3 \%}$ & $\mathbf{6 , 3} \%$ \\
Vesícula palpabIe & $\mathbf{4 9 , 2} \%$ & $\mathbf{2 5 , 3} \%$ \\
Plastrón vesicular & $\mathbf{1 6 , 9 \%}$ & $0,0 \%$ \\
Diarrea & $7,6 \%$ & $5,0 \%$ \\
Signos peritoneales & $10,7 \%$ & $0,0 \%$ \\
Mal cstado general & $\mathbf{1 0 , 7 \%}$ & $0,0 \%$ \\
& & \\
\hline
\end{tabular}

Tabla N: 2

\section{COLECISTITIS $Y$ COLELITIASIS EN NIÑOS}

1962 - 1975 - 231 casos. Hospital Roberto del Río Sintomatologia en menores de 6 años

(12 casos)

\begin{tabular}{lr}
\hline & Porcentaje \\
\hline Dolor abdominal & $100,0 \%$ \\
Vómitos & $66,6 \%$ \\
Fiebre & $71,4 \%$ \\
Jctericia & $33,3 \%$ \\
Mal estado general & $33,3 \%$ \\
Diarrea & $50,0 \%$ \\
Signos peritoneales & $16,6 \%$ \\
Crisis anteriores & $0,0 \%$ \\
Dispepsio & $0,0 \%$ \\
Plastrón vesicular & $0,0 \%$ \\
\hline
\end{tabular}


Tabla N: 3

COLECISTITIS Y COLELITIASIS EN NIÑOS

1962 - $1975-231$ casos. Hospital Roberto del Río

Exámenes de Laboratorio

\begin{tabular}{lcc}
\hline & Col. aguda & Col. crónica \\
\hline Efectuados & $28,5 \%$ & $34,3 \%$ \\
Leucocitosis & $78,2 \%$ & $48,4 \%$ \\
Bilirrubinemia (+ $1 \mathrm{mg})$ & $36,1 \%$ & $30,5 \%$ \\
Transaminasas & $13,8 \%$ & $8,3 \%$ \\
Fosfatasas alcalinas & $0,0 \%$ & $8,3 \%$ \\
Protrombinemia & $2,8 \%$ & $2,8 \%$ \\
\hline
\end{tabular}

Tabla N: 4

\section{COLECISTITIS Y COLELITIASIS EN NIÑOS}

1962 - 1975 - 231 casos. Hospital Roberto del Río

Estudio Radiológico

\begin{tabular}{lrr}
\hline & Agudas & Crónicas \\
\hline & & \\
Total electuado & $21,5 \%$ & $93,6 \%$ \\
Exclusión vesicular & $50, \%$ & $29,7 \%$ \\
Litiasis & $42,8 \%$ & $61,1 \%$ \\
Colangiogralía directa & $7,2 \%$ & $6,5 \%$ \\
Biligrafin & $0,0 \%$ & $2,7 \%$ \\
Falsos positivos & $0,0 \%$ & $2,5 \%$ \\
\hline
\end{tabular}

Tabla N $N^{a} 5$

\section{COLECISTITIS Y COLELITIASIS EN NIÑOS}

$1962-1975-231$ casos. Hospital Roberto del Rio

Tratamiento quirúrgico efectuado

\begin{tabular}{lrr}
\hline & Número & Porcentaje \\
\hline Colecistectomía & 225 & $97,4 \%$ \\
Colecistostomía & 6 & $2,6 \%$ \\
Coledocostomía & 16 & $7,1 \%$ \\
Apendicectomía & 14 & $6,0 \%$ \\
\hline
\end{tabular}

Tabla N: 6

COLECISTITIS Y COLELITIASIS EN NINOS

1962 - 1975 - 231 casos. Hospital Roberto del Río

Hospitalización

\begin{tabular}{lrr}
\hline & Colecistitis aguda & Crónica \\
\hline & & \\
Días preoperatorio & 2,65 & 4,50 \\
Dias pestoperatorio & 7,51 & 5,70 \\
Días de hospitalización & 11,26 & 10,20 \\
\hline
\end{tabular}

Tabla No 7

COLECISTITIS Y COLELITIASIS EN NISOS

1962 - $1975-231$ casos. Hospital Roberto del Río

Conplicaciones

\begin{tabular}{lrr}
\hline & Nümero & Porcentaje \\
\hline Infección herida op. & 10 & $\mathbf{4 , 3} \%$ \\
Respiratorias & 5 & $2,1 \%$ \\
Sección de colédoco & 4 & $1,7 \%$ \\
Fistula biliar externa & 2 & $0,8 \%$ \\
Obstrucción intestinal & & $0,8 \%$ \\
por bridas & 2 & $0,4 \%$ \\
$\begin{array}{l}\text { Estenosis residual } \\
\text { Litiasis residual de }\end{array}$ & 1 & $0,4 \%$ \\
colédoco & 1 & $10,9 \%$ \\
Total & 26 & \\
\hline
\end{tabular}

\section{EXAMENES DE LABORATORIO}

En las colecistitis agudas sólo en algunos casos se efectuaron $(28 \%)$, siendo la leucocitosis un indice constante $(78,2 \%)$. En las colecistitis crónica se hacen con menos frecuencia y sólo en la mitad hay leucocitosis. (Tabla N.9 3).

\section{RADIOLOGIA}

En las colecistitis agudas se efectuó colecistografía en un reducido número de enfermos $(21,5 \%), y$ en estos casos la mitad mostró exclusión vesicular $(50 \%)$ y la otra mitad la presencia de litiasis $(42,8 \%)$. 
En las colecisitis crónicas es un examen de rutina $(93,6 \%)$, llegando una positividad de dos tercios de litiasis $(61,1 \%)$ y un tercio de exclusion vesicular $(29,7 \%)$.

La colangiografía intraoperatoria se hizo cuando se sospechó obstrucción a nivel del colédoco $(7,2 \%$ en agudas y $6,5 \%$ en crónicas). (Tabla $\mathrm{N}^{\circ} 4$ ).

\section{TRATAMIENTO QUIRURGICO}

La colecistectomía, es el tratamiento de elección de las colecistitis $(97,4 \%)$; 6 colecistomías corresponden a operaciones practicadas en los primeros años de esta revisión.

Coledocastomías se realizaron en 16 enfer* mos.

En 14 niños de colecistitis crónica se efectưó apendicectomía electiva. (Tabla № 5 ).

La litiasis vesicular es extraordinariamente frecuente $(86,1 \%)$, correspondiendo a 105 enfermos de colecistitis aguda y 95 con crónica. (Figura No 2).

HOSPITAL ROBÉRTO OEL RIO COLECISTITIS Y COLELITIASIS EN NIÑOS $1962-1975-231$ casos

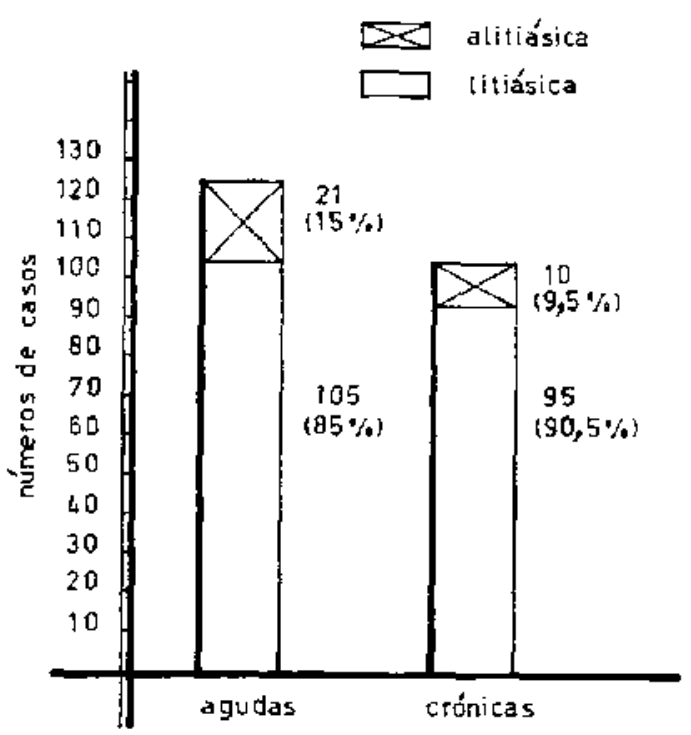

Figura N: 2

La evolución de la intervención quirúrgica en general fue favorable, confirmada por el bajo promedio de días-camas en el posto- peratorio, el bajo número de complicaciones y fa baja mortalidad. (Tabla No 6 ).

Las complicaciones aparecen con mayor frecuencia en las colecistitis agudas, siendo éstas la jnfección de la herida operatoria en 10 casos. Complicaciones graves hubo 4 secciones de colédoco $(1,7 \%)$, que se corrigieron quirúrgicamente, 2 fístulas biljares externas de buena evolución posterior y 2 lesiones residuales, una litiasis y una estenosis. Complicaciones alejadas dos obstrucciones por bridas. (Tabla N! 7).

En nuestra seric hay sólo 2 enfermos fallecidos, por colecistitis agudas con gran compromiso general, signos peritoneates, que se operaron de urgencia $(0,86 \%)$ y fallecieron durante el acto operatorio.

\section{DIAGNOSTICO DE EGRESO}

Del análisis de los diagnósticos de egreso (Tabla $N^{\circ}: 8$ ) se comprueba litiasis vesicular en 263 casos $(87,8 \%)$, en 14 niños se confirma etiología tífica y en 5 casos pancreatitis conconitante.

En un solo caso se comprobó el diagnóstico de anemia hemolítica y en 12 niños hubo parasitosis intestinal. (Tabla № 8 ).

\section{CONCLUSIONES}

Se revisan 251 fichas clínicas correspondientes a enfermos con los diagnósticos de colecistitis agudas y crónicas del Servicio de Cirugía Infantil y Ortopedia del Hospital Roberto del Rio, entre los años 1962 y 1975.

Llama la atención la alta incidencia de esta patología en relación a lo publicado.

En cuanto al sexo hay un franco predominio del femenino: $70,9 \%$, siendo en los menores de 6 años más frecuente el masculino. El grupo etario entre 12 y 15 años ocupa el $76 \%$.

Se analiza la sintomatología clínica de 126 enfermos de colecistitis aguda y de 105 de colecistitis crónica, destacando los signos positivos.

En los exámenes complementarios se men. ciona la importancia y uso de la colecistogra- 
fía y sus resultados. El tratamiento quirúrgico efectuado es la colecistectomía y sólo se realizaron 16 coledocostomía. La litiasis vesicular está presente en un $86.1 \%$ de los operados.

La evolución postoperatoria fue favorable, destacando la baja frecuencia de complicacio nes y un $0,86 \%$ de mortalidad global.

Tabla N: 8

\section{COLECISTITIS Y COLELITIASIS EN NINOS}

1962.1975. 231 casos, Hospital Roberto del Río

Diagnóstico de egreso:

\begin{tabular}{lrr}
\hline & Número & $\%$ \\
\hline Colecistitis ag. litiásica & 101 & 43,7 \\
Colecistitis cr. litiásica & 92 & 39,8 \\
Colecistitis tífica litiásica & 10 & 4,3 \\
Colccistitis túfica alitiásica & 4 & 1,8 \\
Fancreatitis + colelitiasìs & 3 & 1,3 \\
Pancreatitis + colecistitis ag. & 2 & 0,8 \\
Estenosis del cístico & 4 & 1,8 \\
Hipertrofia ganglio cístico & 1 & 0,4 \\
Malformación vesicular-tabique & 1 & 0,4 \\
Anenias liemolítica + colelitiasis & 1 & 0,4 \\
Oxiuriasis & 1 & 0,4 \\
Parasitosis intestinal + colelitiasis & 11 & 4,7 \\
\hline
\end{tabular}

\section{SUMMARY}

Two hundred and fifty one clinical records of patients with the diagnosis of acute Cholecystitis and Cholelithiasis, were received at the Roberto del Rio Children's Hospital between the years 1962-1975.

Of the 251 patients admited for surgical treatment, only 10 of them didn't undergone operator, for abcense of clinical symtons attributable to Cholecystitis. All the patients were under 15 years old, and mos of them in the age gtoup $12-15$ years ( $76 \%$ ) $70.9 \%$ were girls.

The typical picture of Acute Cholecystitis was present in 126 patients, consisting in: severe and acute colic pain, located in the right hypocondrial area. This pain within the hours became permanent. Other important feature were: vorniting $(84.6 \%)$ and fever $(58.4 \%)$.
The physical examination showed an acute pain the right hypocondrial area, with the finding of an hydropic gallbladder in $49.2 \%$ of the cases, and inflamatory mass $16.9 \%$

The diagnosis of Cronic Cholecystitis was made in 105 patients. $14.1 \%$ of the patients had previous biliary colic pain, without fever or malaise.

The main clinical symptons were: acute colic pain in the right hypocondrial area, vomiting (46\%) and hydrops of the gallbladder $(23.3 \%)$. In this patients and $X$ Rays study was possible.

In patients under 6 years old, Acute Cholecystitis was clinically different: They never had previous biliary colic pain, or dyspetic disorders. They came to the Hospital at the first colic pain, with fever vomiting and jaundice. They were severely ill.

The surgical treatment was the same them the others cases. Only 6 patients had an ostomy of the gallbladder the opering of the cholecodus was made in 19 cases. Who had jaundice or palpation of small stones in the duct, during the surgical procedure.

$X$ Rays studies of the ducts during the operation was perfomed in $7 \%$ of the cases.

The findings of stone by pathologist was present in $86.1 \%$ of the cases.

The post operative course was uneventful and short, with a very law mortability rate $(0.86)$.

\section{REFERENCIAS}

1. Aboulola, H. M. et al. Ann. Chir. Infant. 12: 53, 1971.

2. Brenner, $R$. W. and C. Stewart. Rev. Surg. 21: 327, 1964.

3. Dutta, T. et al. Progr. Pediatr. Surg. 8: 109, 1975.

4. Forshall, $I$. and P. P. Rickhom. Brit. J. Surg. 42: $161,1965$.

5. Gibson, J. J. A. M. A. 85: 31, 1925.

6. Gravier, L. et al. Surgery 63: 696, 1958.

7. Morales, L, et al. Y. Pediatr. Sur. 0: 565, 1967.

8. Ramirez, S. y otros. Bol. Méd. Hosp. Inf. Méxíco. 29: 293. 1972.

9. Scobie, $W$. and Bentley 7. J. Pediatr. Surg, 4: 457,1969 .

10. Swenson, $O$. Pediatric Surgery. 3* ed. New York. Appleton Century Crofts. 1969.

11. Ulin, A. and I. L. Nasa. Surgery 31: 312, 1952. 